\title{
A MOBILE INTELLIGENT SYNTHETIC CHARACTER WITH NATURAL BEHAVIOR GENERATION
}

\author{
Jongwon Yoon and Sung-Bae Cho \\ Department of Computer Science, Yonsei University, Seoul, Korea \\ jwyoon@sclab.yonsei.ac.kr,sbcho@cs.yonsei.ac.kr
}

Keywords: Intelligent synthetic character, Intelligent agent, Smartphones.

\begin{abstract}
As cell phones have become essential tools for human communication and especially smartphones rise as suitable devices to implement ubiquitous computing, personalized intelligent services in smartphones are required. There are many researches to implement services, and an intelligent synthetic character is one of them. This paper proposes a structure of emotional intelligent synthetic character which generates natural and flexible behaviour in various situations. In order to generate the character's more natural behaviour, we used the Bayesian networks to infer the user's states and we used OCC model to create the character's emotion. After inferring these information, the behaviours are generated through the behaviour networks with using the information. Moreover, we organized a usability test to verify a usability of the proposed structure of the character.
\end{abstract}

\section{INTRODUCTION}

Recently, cell phones appear to play an indispensable role for human communication. Various additional services using mobile networks have been developed and many enterprises have competitively come out with high-end devices, especially smartphones, which have both functions of a PDA (Personal Digital Assistant) and a cell phone due to not only an expansion of subscribers but also an improvement of transmission speed. Moreover, as smartphones become the device suitable for realizing ubiquitous computing environments, a necessity of personalized intelligent services for smartphones is getting increase.

The intelligent synthetic character is an autonomous agent which behaves based on its own internal states, and can interact with a person in realtime. The intelligent synthetic character can be applied to entertainment robot and service robot (Kim et al., 2002).

There is an issue when implementing the intelligent synthetic character, that is to make the character seem to alive. To achieve this successfully, the character should express various behaviors naturally and flexibly to make user feel that the character behaves with its consciousness, not with some simple rules for generating behaviors.
In this paper, we propose a method of generating behaviors of the synthetic character. The Bayesian networks are used to infer user's states and OCC model is used to generate the character's emotions. Moreover, we use behavior networks with information gathered from previous process to generate character's natural and flexible behaviors.

\section{RELATED WORKS}

Researches about the intelligent service on mobile devices are mostly focused on the method to provide personalized services using contexts around users and smartphones. S. Schiaffino et al. (Schiaffino et al., 2002) designed the software structure of the personalized schedule manager agent, and proposed a case-based reasoning method using the Bayesian networks. And Kim et al. (Kim et al., 2004) proposed an intelligent agent that selects the behavior using its internal state and learns with the interaction with user. Marsa-Maestre et al. (MarsaMaestre et al., 2008) proposed architecture of the mobile agents which is applicable to smart environments for service personalization. And Sung et al. (Sung et al., 2008) designed an intelligent agent that infers users' status with case-based learning. 
Considering these points, we designed the mobile intelligent synthetic character which recognizes external situations and behaves intelligently. To achieve this, we used the emotion model to create the emotional state of the character and the behavior network to generate the character's natural and flexible behaviors with its own emotional state.

\section{INTELLIGENT SYNTHETIC CHARACTER}

The intelligent synthetic character consists of four components: perception system, emotion system, motivation system and behavior generation system. Figure 1 shows an architecture of the intelligent synthetic character.
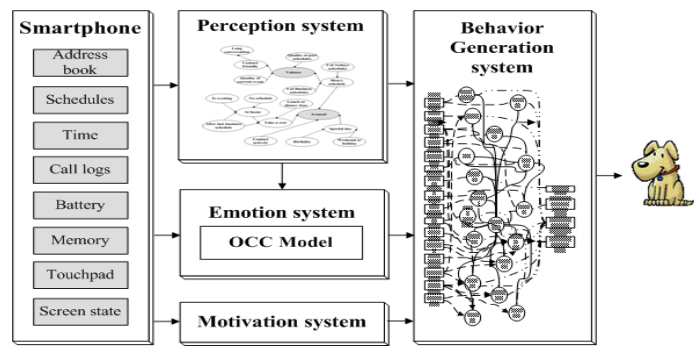

Figure 1: An architecture of an intelligent synthetic character.

\subsection{Perception System}

The perception system gathers information that is available in smartphones such as contacts information, schedules, call logs, and device states. Moreover, the perception system reasons the states of the user. In this paper, the character infers especially the user's valence and arousal state, and the business state.

The Valence-Arousal(V-A) model is a simple model that represents emotion as a given position in a two-dimensional space. It has been commonly used in previous studies on emotion recognition (Picard, 1995). However, we do not deal with user's exact emotions directly mapped on the V-A space such as happy, sad, fear, etc. because it's hard to recognize the user's exact emotional state based on the simple contexts in the smartphones. The system reasons roughly the user's valence and arousal states.

The perception system uses Bayesian networks as a context-reasoning method of its own. The Bayesian network is a representative method to infer states with insufficient information and uncertain situations. We designed the Bayesian network which infers the user's valence, arousal, and business states. Figure 2 shows a part of the Bayesian networks we designed.

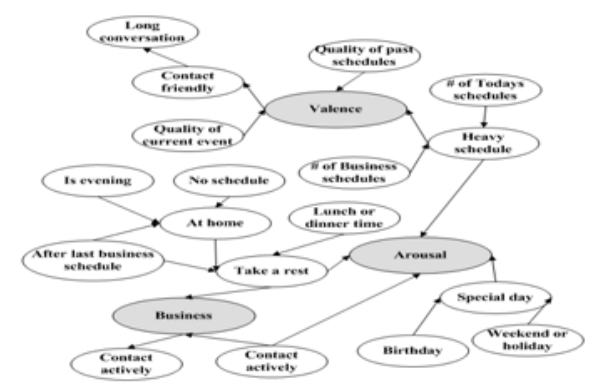

Figure 2: A part of the Bayesian networks.

\subsection{Emotion System}

Emotions are particularly important for characters, because they are an essential part of the selfrevelation feature of messages (Bartneck, 2002). The emotion system uses OCC model (Ortony et al., 1988) to create the character's affections among various emotion models.

The OCC model is the standard model proposed for a synthesis of emotions, and that is based on cognitive appraisal theory of emotion. In this paper, we modified the original OCC model and proposed the modified OCC model which has 14 emotional categories. Figure 3 shows the proposed modified OCC model. The emotion that has the highest intensity among whole categories is chosen as the current emotion of the character.

\subsection{Motivation System}

Goal also takes an important part of the intelligent synthetic character. Like human, the character behaves with its own goals which motivate it. This makes the character more realistic. The character does not behave with fixed goals but with the goals that can be changed according to the current situation.

For examples, when the battery is low, the motivation system sets the goal as 'Be in agreement with phone state' to warn that the battery is low to user.

\subsection{Behavior Generation System}

In the behavior generation system, the behavior network (Maes, 1989) is used as the method for selecting the most natural and suitable behavior for the situations. The behavior networks are the model that consists of relationships between behaviors, 


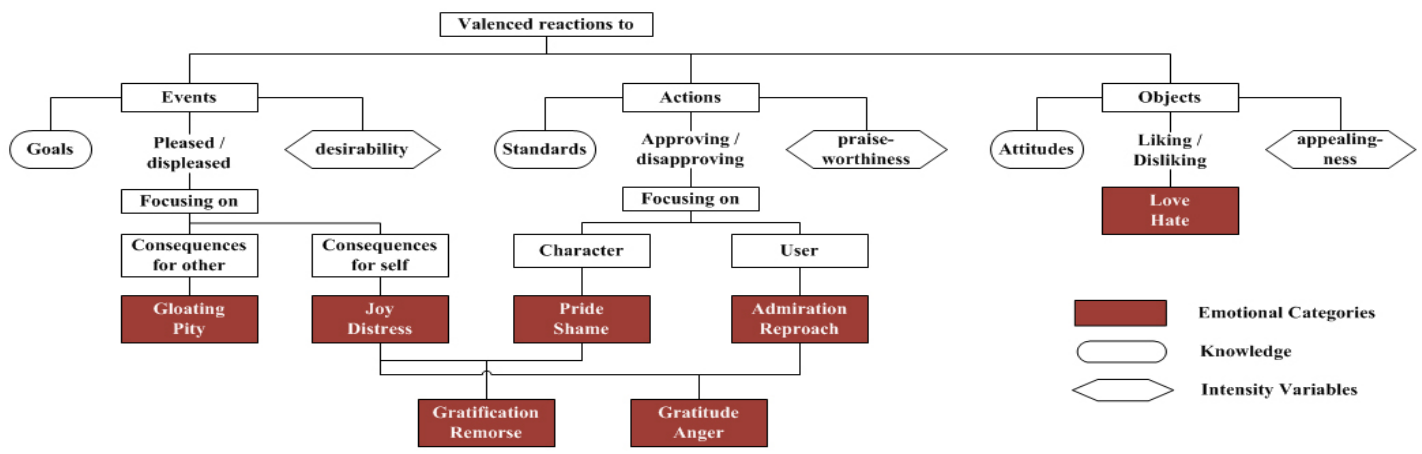

Figure 3: Modified OCC Model.

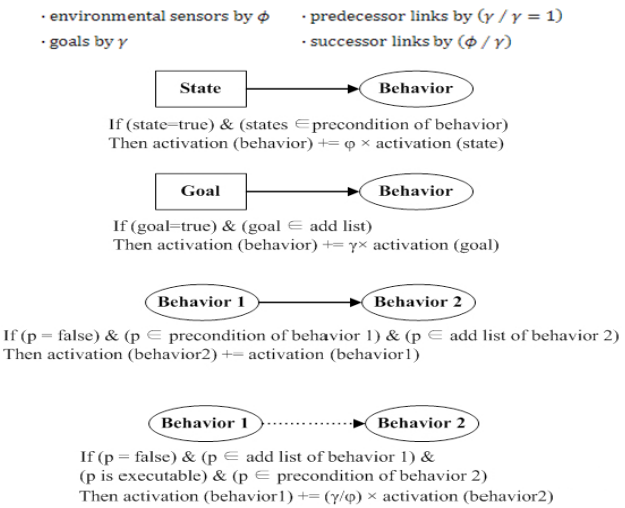

Figure 4: A part of the behaviour network.

goals, and external environment, and selects the most suitable behavior for the current situation. Behaviors, external goals and internal goals are connected with each other through links.

The behavior network selects the behavior which has the highest activation energy. The activation energy is a degree which represents how much the behavior is activated. Figure 5 shows spreading of activation with the following weights.

In this paper, the proposed behavior generation networks generate 31 behaviors using the conditions such as the user's states, the character's emotion, the user's current behavior and the current time, and its goals set by the motivation system. Figure 4 shows a part of the behavior generation networks designed.

\section{IMPLEMENTATION}

To develop the proposed intelligent synthetic character, we used the Microsoft Visual Studio 2008 and the Microsoft Windows Mobile 5.0 SDK, and the character actually runs on the Samsung SPHM4650 smartphone. Figure 6 shows screen shots of the character.

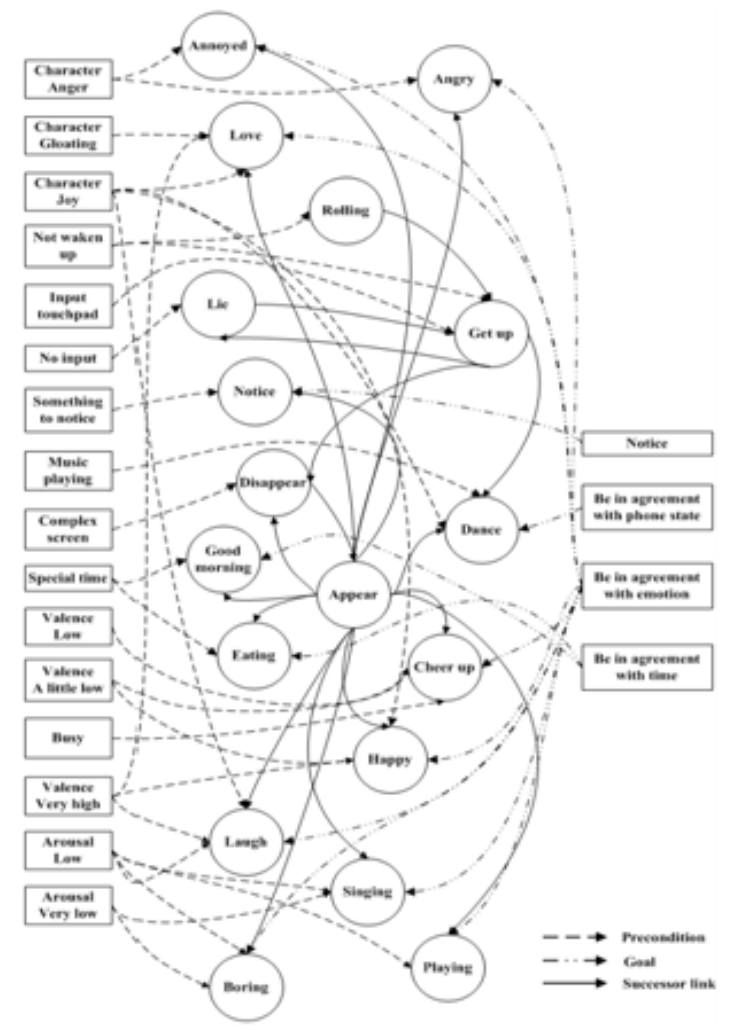

Figure 5: Spreading activations ( $\mathrm{p}$ is conditions of the current state).

\section{USABILITY TEST}

In order to evaluate the fitness of the behaviors generated through the proposed method on various situations, we performed the usability test as follows. Eight participants in their twenties were recruited for the test. Most of participants reported that they heard about the intelligent character but none of participants had used it in actual. In the usability test, the participants carried out 10 scenarios. 


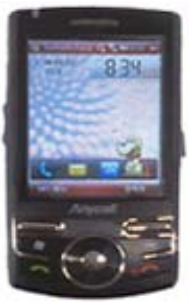

(a)

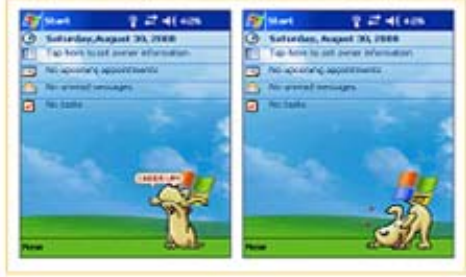

(b)
Figure 6: Screen shots of an intelligent synthetic character on the actual smartphone(a) and the simulator(b).

The participants observed a sequence of five behaviors for each scenario generated randomly and another sequence of 5 behaviors generated by the proposed method, respectively. The random generation method just selects any behaviors randomly to the user without understanding situations

After the previous task, the participants evaluated the fitness of each behavior on each situation. The fitness ranged from 1 which means "Strongly incongruent" to 5 which means "Strongly suitable." Following the evaluations, we summed up the fitness scores of each participant distinguishing two different behaviors generating methods, randomly generating method and the proposed method, and measured the average fitness scores of each method. Table 1 shows the average fitness scores evaluated by the participants.

In order to analyze the result of the usability test, we conducted the Wilcoxon signed-rank test with the fitness scores. As the result of the Wilcoxon signed-rank test, we got P-value by 0.012 , thereby we accept the alternative hypothesis because P-value is smaller than 0.05 . It confirms that the proposed method is appropriate to generate the character's behaviors.

\section{CONCLUDING REMARKS}

We presented the architecture of the mobile intelligent synthetic character for its natural behaviors. In order to provide enhanced intelligent services, it is necessary for the intelligent synthetic character to interact with the user and evolve by itself. To achieve this, we will attempt to develop algorithms for an interaction and an evolution, especially the learning system that evolves the structure of the Bayesian networks and the behavior generation network with the user's feedback in the future work.
Table 1: Average fitness scores.

\begin{tabular}{|c|c|c|}
\hline Participants & Random generation & Proposed method \\
\hline 1 & 2.46 & 4.50 \\
\hline 2 & 2.40 & 4.22 \\
\hline 3 & 3.00 & 4.44 \\
\hline 4 & 2.14 & 4.38 \\
\hline 5 & 2.44 & 4.40 \\
\hline 6 & 3.12 & 4.44 \\
\hline 7 & 2.88 & 4.54 \\
\hline 8 & 2.06 & 4.20 \\
\hline
\end{tabular}

\section{ACKNOWLEDGEMENTS}

This work was supported by the IT R\&D program of MKE/KEIT (10033807, Development of context awareness based on self learning for multiple sensors cooperation).

\section{REFERENCES}

Y.-D. Kim, Y.-J. Kim, J.-H. Kim and J.-R. Lim, "Implementation of artificial creature based on interactive learning," In Proc. of 2002 FIRA Robot World Congress, pp. 369-373, 2002.

S. Schiaffino and A. Amandi, "On the design of a software secretary," In Proc. of the Argentine Symp. on Artificial Intelligence, pp. 218-230, 2002.

Y.-D. Kim, J.-H. Kim and Y.-J. Kim, "Behavior generation and learning for synthetic character," Evolutionary Computation, vol. 1, pp. 898-903, 2004.

I. Marsa-Maestre, M. A. Lopez-Carmona, J. R. Velasco and A. Navarro, "Mobile agent for service personalization in smart environments," Journal of Networks, vol. 3, no. 5, pp. 30-41, 2008.

B.-K. Sung, "An intelligent agent for inferring user status on smartphone,” Journal of KIIT, vol. 6, no. 1, pp. 5763, 2008.

R. Picard, "Affective Computing," Media Laboratory Perceptual Computing TR 321, MIT Media Laboratory, 1995.

C. Bartneck, "Integrating the OCC model of emotions in embodied characters," In Proc. of the Workshop on Virtual Conversational Characters: Applications, Methods, and Research Challenges, 2002.

A. Ortony, G. Clore, and A. Collins, The Cognitive Structure of Emotions, Cambridge University Press, Cambridge, UK, 1988.

D.-W. Lee, H.-S. Kim and H.-G. Lee, "Research trend on emotional communication robot," Journal of Korea Information Science Society, vol. 26, no. 4, pp. 65-72, 2008.

P. Maes, "How to do the right thing," Connection Science Journal, vol. 1, no. 3, pp. 291-323, 1989. 